\title{
Adenoid Cystic Carcinoma (ACC) of the Breast: Unusual Breast Pathology in Young Women
}

\section{Díaz Ortega Patricia*, Osinaga Alcaraz Maite, García Manero Manuel, Segura Ortega Vanesa, Bezares Pérez Blanca and Ochoa Prat Anabel}

Department of Gynecology and Obstetrics, Hospital García Orcoyen, Estella, Spain

*Corresponding Author: Díaz Ortega Patricia, Department of Gynecology and Obstetrics, Hospital García Orcoyen, Estella, Spain.
Received: January 13, 2020

Published: January 29, 2020

(C) All rights are reserved by Díaz Ortega

Patricia., et al.

DOI: 10.31080/ASWH.2020.02.0086

\begin{abstract}
Introduction: Adenoid cystic breast carcinoma is a tumour of extremely rare malignant behavior, belonging to the group of triple negative breast tumors but, unlike these, has a favorable prognosis with high survival if the diagnosis and management are appropriate $[1,7,9]$.

Presentation of Case: A 34 year old female patient presented symptom is a well-circumscribed palpable mass in the right breast with recurrent breast pain that not improve with analgesic or progestin gel. Excision biopsy of a lump in the upper outer quadrant revealed adenoid cystic carcinoma. A right sided lumpectomy was performed, with sentinel node biopsy. Histopathology revealed a fibroadenoma accounting for one mass and adenoid cystic carcinoma in another, confirmed on Periodic acid Schiff (PAS) staining, with a very closely margin. A second lumpectomy was performed with focal adenoid cystic carcinoma. Finally a right side modified radical mastectomy (MRM) was performed. The sentinel node biopsy was negative. There was no evidence of recurrence or distant spread at follow-up 12 months postoperatively.

Discussion: Adenoid cystic carcinoma of the breast is a rare tumor, of which very few cases have been described in the literature and which lacks in-depth studies on its diagnosis and management due to its rarity [1,2]. Nowadays, its diagnosis is based on biopsy excision and anatomopathological study. And the management, like the Basal_Like tumors of the breast, is based on surgical excision with a wide margin of safety. However, unlike these, axillary nodal study is not justified, and the use of posterior adjuvant is controversial with various opinions in this regard [1,3].

Conclusion: ACC is a rare malignant neoplasm. Surgical treatment is the mainstay with little role for radiotherapy and chemotherapy according to the published literature.
\end{abstract}

Keywords: Adenoid Cystic Carcinoma; Ultrasound; Modified Radical Mastectomy

\section{Abbreviation}

ACC: Adenoid Cystic Carcinoma; ER: Strogen Receptor; PR: Progesterone Receptor.

\section{Introduction}

Adenoid cystic carcinoma of the breast is a rare tumour with basal-likely characteristics, hormone receptor negative and ERB2 negative $[1,2]$. Despite these characteristics, its behaviour is of low degree of malignancy; it rarely presents nodal spread or distant metastasis; for which the global survival and disease-free survival reaches $90 \%$.

It is due to its rarity, so there are hardly any studies or consensus about its diagnosis and clinical management.
Nowadays, diagnosis is based on the anatomopathological study of samples obtained by fine needle puncture. It presents a very characteristic histology [4].

The treatment consists of the excision of the piece with a wide safety margin. The use of adjuvant therapy is controversial, with variable opinions on the subject; however, it has been seen that none of the treatments, chemotherapy, radiotherapy or hormone therapy, modify long-term survival $[1,6]$.

We present the case of a 34-year-old patient with FIGO stage T1N0M0 adenoid cystic carcinoma; we discuss the diagnosis and clinical management of this entity by reviewing the literature. 


\section{Case Report}

A 34 year old female presented symptom in a well-circumscribed palpable mass in the right breast with recurrent breast pain that not improve with analgesic or progestin gel. Excision biopsy of a lump in the upper outer quadrant revealed adenoid cystic carcinoma. A right sided lumpectomy was performed, with sentinel node biopsy.. There was no past history of breast surgery or breast irradiation. On examination three painless, freely mobile lumps were palpable, up to $3 \mathrm{~cm}$ in maximal diameter, making the right breast larger than the left. The skin of both breasts was normal. Right sided axillary lymph nodes were not palpable. Mammography suggestive of right breast as multiple, irregular, hyperdense mass lesion diffusely present in the breast, largest measuring approximately $19 \mathrm{~mm}$ with irregular speculated margins causing distortion of adjacent parenchyma. Ultrasonography suggestive oval mass in right breast largest measuring about $2 \times 1.5 \mathrm{~cm}$ possibility of malignant lesion (Figure 1).

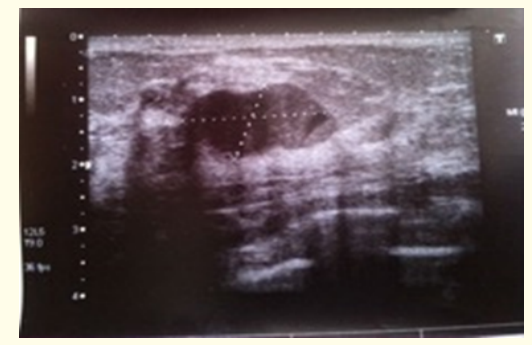

Figure 1: Breast ultrasound. An irregular isoechogenic nodule suggestive of malignancy is observed.

Fine needle aspiration cytology of one mass was unconclusive. . Excision biopsy of another right breast lump was diagnosed as adenoid cystic carcinoma with negative ER,PR and HER2; and low expression of Ki67 (Figure 2). Right modified radical mastectomy was performed with sentinel node biopsy which one was negative for malignancy. The histopathology was consistent with residual ACC (confirmed on PAS staining), with the surgical margins free from malignant cells. Patient did not receive any adyuvant treatment. There was no evidence of recurrence or distant spread at follow-up 12 months postoperatively.

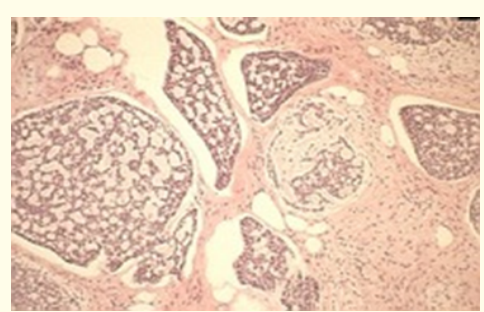

Figure 2: Histological appearance to hematoxylin-eosin staining: mucus pools surrounded by epithelial cells with a small cytoplasm and hyperchromatic nucleus.

\section{Discussion and Conclusion}

Adenoid cystic carcinoma of the breast is an extremely rare malignant tumour, accounting for $0.1 \%$ of malignant tumours of the breast according to the series [1]. This type of tumor is characteristic of the salivary glands, but can be found in other locations such as the lung, breast or prostate. In all cases their behaviour is low grade malignant.

As for the ACC of the breast, it belongs to the group of Triple Negative or Basal Like tumours, since it presents negativity for ER, RP and HER2; however, and unlike Basal Like tumours, it does not present somatic mutations in p53,PIK3CA, RB1, BRCA1 and BRCA2; It is characterized by a great heterogeneity of mutations, the most characteristic being the $t(6 ; 9)(q 22-23 ; p 23-24)$ translocation that results in the MYB-NFIB gene [2]. This gene has been located in 23$100 \%$ of breast CCAs and could be considered a therapeutic target in the future.

Its histological appearance to hematoxylin-eosin staining is very characteristic, presenting mucus pools surrounded by epithelial cells with a small cytoplasm and hyperchromatic nucleus [5].

It is a tumor that appears in older women, although cases in men have also been described, associated or not with gynecomastia. In our case, the patient was 34 years old, and had no personal or family history of breast pathology or previous radiation; which makes the case even more exceptional.

It has an indolent clinical course, presenting itself as a palpable nodule in most cases, which implies an advanced stage to diagnosis, according to the FIGO classification. In addition to this classification, Rosen et al [8] classify this tumour in 3 grades according to the solid component (Grade 1: completely cystic tumour; Grade 2: $<30 \%$ solid component; Grade 3: $>30 \%$ solid component) so that its malignant behaviour will also depend on this characteristic.

Given the infrequency of this tumor, there is no consensus on its diagnosis and clinical management [1,9]. Fine needle aspiration, or vacuum needle aspiration would be the techniques of choice for histological diagnosis; although in some cases, such as ours, the result is inconclusive and an excision-biopsy is required for correct typing.

There is also no consensus on which imaging technique is best when locating the lesion and planning surgical treatment. There have been discordant results regarding the size of the lesion between MRI and ultrasound or mammography [4]; this makes a careful radiological study by trained personnel necessary. 
Today, surgical treatment is the treatment of choice for these tumors. A wide excision with a negative margin is crucial. Since it is a tumor that does not usually spread by nodal route, the presence of nodal metastases is less than 5\%, even in larger tumors; therefore, an axillary nodal clearance would not be indicated initially. Regarding the study of the sentinel node, there is controversy, perhaps because there are few studies on this subject due to the rarity of this tumor. In the review by Treitl et al, carried out in a centre with 20 years of experience in the treatment of these tumours, they suggest that the study of the sentinel node should not be carried out systematically, as its results would not influence overall survival or disease-free survival.

Unlike other Triple-Negative tumors, it is not a chemosensitive or radiosensitive tumor, and the role of hormone therapy is also controversial. There is great controversy regarding the benefit of using adjuvant therapy and its impact on the survival of these patients. However, it is a tumor with a good overall prognosis, with 10 -year survival rates close to $90 \%$. Only $2 \%$ of patients present metastatic disease at diagnosis and the risk of local recurrence is less than $20 \%[1,9]$.

We conclude that ACC of the breast is an extremely rare tumor with basal-likely characteristics but, unlike this type of tumor, has a favorable prognosis even in young women, if a correct diagnosis and proper management is made.

\section{Bibliography}

1. Treitl., et al. "Adenoid cystic carcinoma of the breast, 20 years of experience in a single center with review of literature". Breast Cancer 25 (2018): 28-33.

2. Martelotto., et al. "Genomic landscape of adenoid cystic carcinoma of the breast". Journal of Pathology 237.2 (2015): 179189.

3. Kashiwagi., et al. "Adenoid cystic carcinoma of the breast: a case report". Case Reports in Oncology 12 (2019): 698-703.

4. Agafonoff., et al. "Adenoid cystic carcinoma of the breast-Discordant size on imaging and pathology: a case report and review of literature". Annals of Medicine and Surgery (Lond). 43 (2019): 1-4.

5. JG Azzopardi. "Problems in breast pathology". WB Saunders, Philadelphia, PA (1979): 335-359.

6. M Culubret and I Roig. "Fine needle aspiration biopsy of adenoid cystic carcinoma of the breast: a case report". Diagnostic Cytopathology 15.50 (1996): 431-434.
7. MW Stanely., et al. "Adenoid cystic carcinoma of the breast: diagnosis by fine needle aspiration". Diagnostic Cytopathology 9.2 (1993): 184-187.

8. Rosen., et al. "Adenoid cystic carcinoma of the breast, a morphologically heterogenous neoplasm". Pathology Annual (1989): 237-254.

9. PP Anthony and PD James. "Adenoid cystic carcinoma of the breast: prevalence, diagnostic criteria, and histogenesis". Journal of Clinical Pathology 28.8 (1975): 647-655.

\section{Assets from publication with us}

- Prompt Acknowledgement after receiving the article

- Thorough Double blinded peer review

- Rapid Publication

- Issue of Publication Certificate

- High visibility of your Published work

Website: https://www.actascientific.com/

Submit Article: https://www.actascientific.com/submission.php

Email us: editor@actascientific.com

Contact us: +919182824667 